\title{
Assessment of Eye-hand Coordination in Italian Preschools
}

\author{
Ambretti Antinea,Viscione Ilaria, D’Elia Francesca \\ Department of Human, Philosophical and Educational Sciences, University of Salerno, Fisciano Salerno 84084, Italy
}

\begin{abstract}
In 2012, the Italian Ministry of Education introduced in the Guidelines for preschool the need to evaluate one aspect of coordination related to the eye-hand relationship. Meinel's theoretical model provides an overview of the eye hand coordination, based on capacity to integrate anatomic-functional skills with specific and synchronic hand's actions. Meinel's model is rich of possible teaching ideas which are consistent with the Italian school system. They might also be a topical theoretical support to choose and use the motor evaluation tests in the Italian preschool, in order to evaluate the eye hand coordination and its constituent elements.
\end{abstract}

Key words: Eye-hand coordination, pre-school, assessment.

\section{Introduction}

In the Guidelines for Italian preschool, evaluation of coordination is an important aspect for the eye-hand relationship [1]. More specifically, the ability of integrating the functional anatomic skills of the eye with the specific and synchronic actions of the hand, a subject already evaluated and studied by different evaluating bodies. The coordination of eye-hand movements characterizes the evolution and development phases by which the child is able to coordinate the information gathered through the different sensorial channels [2]. This skill, which requires the efficient use of the hand in relation to the visual parameters, has been defined by Taylor Kulp M., Sortor as the result of the integration of visual information with the movements of the hand [3]. In Italy, Maria Montessori had theorized the cognitive and functional potentiality of the sensory-motor experiences which specifically involve the visual channel in order to enter into a special relationship with the environment [4], thus anticipating the role of the eye-hand coordination function in accessing the educational processes.

Corresponding author: Ambretti Antinea, Ph.D., professor, research field: evaluation test in educational motor contexts.
Therefore, the eye-hand synchrony is the expression of the constant cognitive and social integration which characterizes the motor system and notably the part given to manipulation [5]. The performance of accurate and increasingly practical movements is determined by a complex system of interactions between visual and proprioceptive information, aimed at planning the movements of the hand according to the personal ability to visualize the movements to be performed and to give them a sequence with a direction related to the identification of the position of one's body parts [6]. In goal-directed actions, the hands and eyes of the actor are tightly coordinated both temporally and spatially, and thus, in contexts including manual engagement with objects, hand movements and eye movements provide redundant information about where the eyes are looking.

According to Vayer, practical actions like throwing, catching and drawing, are not only the expression of a highly complex interaction between eyes and hands [9], but also the demonstration of the possible functional use of tactile and visual sensations to interact efficiently with the environment. Psychomotor vision, thanks to one of its most eminent interpreters, Le Boulch, attempts to provide further evaluation keys for eye-hand coordination considering 
it as a sort of progressive adjustment of the eye-hand reflex which, following an eye-saccadic movement, enables the projection of the hand on the trajectory of a moving object [8].

Gardner's recent studies on multiple intelligences analysed the intellectual aspect of the eye-hand relationship, which besides satisfying the growing need to efficiently manipulate the reality of objects, people and spaces, represents one of the possible expressions of the body-kinaesthetic aspect of intelligence [9]. From the neurological-physiological point of view, Berthoz has shown that the integrated eye-hand use is fully achieved in the relationship between hand and object and that it expresses a successful level of congruence between the motor selection of a specific type of grasping and the visual skill applied to objects that although of different shape and size, at a motor level share the same encoded grasp [10]. It also measures the organization of the motor system control [10].

For Berthoz, the eye-hand coordination, related to the complexity of coordination tasks [10] involving the entire body and its segments in an endless regulatory activity—is to be considered as the need to realize multiple references connected to the different parts of the body enabling the synergy between the tactile and the visual elements controlling the movements of the arms and of the legs.

\section{The Relevance of Meinel's Theoretical Model in the Italian School System}

Every study on motor coordination, as it includes research into the subject's motor-qualitative skills, necessarily needs to be connected and contextualized within the different phases of the person's growth and development. The ability to control and harmonize one or more movements, in terms both of efficiency and of continuity, is in fact related to a number of factors determined also by the specific phase of growth, maturity and development of the various systems that control the movement, as well as by the subject's physical and psychological differences and the characteristics of his/her cognitive system. Therefore, studies on motor coordination from infancy to pre-adolescence should take into account the various phases of development and the different functionality of the qualitative systems connected to the control of movement, as opposed to investigations which include phases of growth such as adolescence and youth.

Infancy and preadolescence are characterized by the presence of a series of different options and solutions [5] to carry out a combination of movements. However, they also show, at the same time, the impossibility to execute many complex combinations required in contexts that are far more difficult than what a child can control for years [5]. Early childhood, defined by Gesell as the acrobat age, is, for instance, characterized by a great number of movements which represent attempts at a deviated motor activity that the child produces so that his body has a continuous relationship with the outside world. According to Bloch, the child's ability to select and choose movements during his childhood depends on a functional association between sensorial and motor systems that mutually integrate in a final action [2] which amounts to a proper motor programme, characterized by a continuous hierarchy of structures [2]. The child's movement—considered in its different performances, is in fact the result of an adaptation based on a balance between adjustment and a recognizable assimilation with actions coordination enabling him to act on the environment and to transform it with essential results [2].

In this regard, Meinel explains that the requirements for coordinated action are reflected in the functional capacity (functional maturity) of the organs controlling movement [2] to gather, elaborate and transmit information taken from the environment and develop one's functionality in relation to different experiences. The quality of such experiences depends on morphological and functional requirements 
existing in the body and its organs' systems [2, 5] and its feedback.

In this sense infancy, characterized by the specific stages of functional maturity and psycho-motor development, is represented as a time when only a part of the motor skills can be performed since each motor execution is connected with the capacity of motor control. Coordination seems to depend both on the development phase and on the function of the environment in providing the subject with the executive and the building opportunities of motor expertise. Environmental feedback could, in fact, play a key role in defining coordinated actions since the shortage of motor experiences could prolong and complicate a learning process [5]. In the first phase of school age, according to Meinel, the increase in the development of the control skills and combination of movements [2] is tightly connected to the number of experiences the child has lived through already in early infancy. In other words, such experiences could bear an impact capable of influencing the structuring of the coordinative skills and, above-all, of the eye-hand coordination. The clumsy movements, typical of the coarse coordination of early infancy, gradually evolve thanks to the regulatory influence [5] of the environment enabling the child to act with increasing precision, encouraging not just the automatic repetition of a movement [2], but a personal elaboration of sensory data. In child growth, the development of movements, that become ever more precise and functional to achieve a specific action, occurs progressively thanks to the acquisition of knowledge related to the sensory organs. Meinel says that a child's relationship with the real world is provided, to start with, by the synergy between the functionality of the visual channel and the movement of the body and its parts. According to this author, the coordination of the movements of the eyes with that of the hands is pivotal in creating, on the basis of sensations and perceptions, the conditions for the full development of the cognitive processes.
Manipulation, in fact, as it involves the visual-tactile channels, ensures the gradual knowledge of the objective (touchable) existence, of the nature of the material world through the collaboration between motor and sense organs [2, 4]. In this theoretical-experimental approach, also Leontiev has highlighted the significance of the interdependence between sight and motor actions, underlining that the evolution of higher mental processes begins with learning how to manipulate external objects [2]. The gradual improvement of the early sudden and clumsy grasping movements $[2,6,8]$, according to this author, would be related to anatomic functional and cognitive developments which can evolve only on the basis a great number of sensations and perceptions [2]. The integration of the combined movements of the eyes and of the hand directed at manipulation, therefore, would point at a motor action related to an object that cannot be considered as a simple body movement [2, 4], as a consequence the development of the intellectual processes should be considered as the transformation of processes that originally were directed at external objects in the form of motor actions [2, 4, 8].

According to Leontiev, the establishment of the first intentional movements represents a new type of vital relationships [2] that children develop in order to interact with the reality surrounding them. The child achieves his sensory knowledge of things and the reflexion of the objective reality [2] through numerous motor movements and hand-tactile manipulations [2].

Also Dewey's scientific work points to a strong connection between eye-hand movement and cognitive development where the learning by doing concept reveals the natural relationship between action and cognitive processes.

Also Gesell shares the vision that the ability of controlling, adapting and transforming one's movements is already defined at birth by a rigorous evolution process which follows biologically defined stages. However, Meinel states that the representation 
of the world of perceptions mediated by the harmonic use of movements and the visual channel seems to be essential to perform a whole set of motor activities to develop the motor coordination of many types of movement [2].

According to the author, in fact, the first stage of early infancy is the time when the acquisition of multi-purpose movements [2] takes place. The further refinement of these can be considered as the quality improvement of movement progress as well as the increase in the availability of varied movements [2] which would correspond to the development of controlling skills and movements combinations $[2,10]$. The difference among the various type of movements, according to Meinel, would be the first obvious sign of a rapid increase of the motor learning skills [2] connected to the child's characteristics.

From this point of view, the role of physical education in the schools [2] is crucial for children's motor development as it favours the growth of specific peculiarities of motor development already at a very early school age [2].

Meinel indicates, as the precondition of the eye-hand motor activity, the increased ability to concentrate and the transition from a perception that is mainly globally oriented to a highly analytical one focused on details [2], which is the requirement for the development of the eye-hand combination movements. The development of the eye-hand coordination, according to Meinel, is connected to the transition of a large part of functions previously performed by the visual analyzer to the kinaesthetic analyzers [2] and is defined by the experiences that accompany the child in his everyday life and at school.

Thus in accordance with these theoretical-argumentative observations that nowadays are fully acknowledged by the literature dealing with teaching methods of physical education [2], the 2012 National Guidelines for the Italian Preschool Curriculum, contains a clear reference to the importance of planning school activities to help the child to develop his skills of perception, analysis and selection of data gathered by the analyzing organs [1], respecting the individual level of bio-psychological maturity $[1,2,8]$, underlining the functional role for the development of the coordination skills.

Following Meinel's suggestions, the 2012 National Guidelines for the Italian pre-school curriculum, draw attention to the fact that the motor skills needed to change and adapt one's movements in children at this age are still very limited and just beginning to develop $[1,2]$. Therefore it is essential to make sure that the choice of the teaching methodologies, tools and programmes to be adopted, in observance of the plurality of the methodological options, is made in freedom and in an organized manner [1], in order to ensure that the educational targets recommended by the Italian Ministry of Education are met.

Based on Meinel's considerations, the school activities rich in stimuli and guided activities included in the Italian pre-school curricula, require careful planning so as to be able to gather, in a regular and constant way, all the information concerning the development of the models of knowledge and abilities, the willingness to learn, the growth of each student's self-awareness [1, 2], in order to carry out a positive comparison of the different levels of growth both individual and collective [1, 2], choosing and selecting methods and tools of assessment that are different and always attaining to the type of activity considered [2]. We also wish to highlight the importance of the observation tools of the motor capacity as the regular observations carried out by teachers in the course of their didactic activity will be the privileged tool for the constant adaptation of the teaching programmes [1, 2], thus enabling Italian teachers to introduce changes or integrations. The recent 2012 National Guidelines for the curricula intended for primary and pre-schools, require teachers of both school levels to acquire a high level of competence and responsibility of assessment which becomes a leading educational function and, together with the learning processes, will serve as 
motivational tool towards one's progressive improvement [1, 2], stressing the importance of the evaluations made before, during and after the curricular activities [1]. On this subject, Meinel suggests that the goals, tools and methods of the teachers [1] should be clear and suitable for the age, the motor experiences and the knowledge of their pupils [1]. The vision of the evaluation contained in the 2012 National Guidelines of the Ministry of Education conforms to Meinel's [1, 2] interpretation and underlines the importance of steering the planning of educational projects starting with the assessment of the motor development of each individual. In Meinel's opinion, functional maturity depends on the integration of the functional capacity of the movement controlling organs, which are the central nervous system and the sense organs [1] with the motor experiences already acquired by the student and registered in his motor memory [1, 2]. Consequently, the role of the educational action of the teaching person is vital for the definition and selection of the learning goals and the development of new movements [1, 2] starting with the analysis of the motor requirements which will affect the coordination capacity and the existing movement skills [2].

In fact, environments such as family, school, and community play crucial roles on the children's development of movement skills, specifically when these skills are appropriately practiced [11]. Appropriate practices are considered activities designed based on children's developmental levels [12]. The engagement in appropriate practices of movement skills during early childhood results in children's confidence and competence to participate in physical activity [13].

In this sense, the possibility to use a battery of tests for motor evaluation in the schools enables the teachers to gather essential information on the initial motor level of the students with the purpose of acquiring objective data on significant circumstances for the performance diagnosis [2] of the students thanks to standardized measuring and analysis procedures [1, 2] of the maturity levels reached in order to plan educational activities coherent with the initial levels.

In other words, the selection and the use of rigorous scientific evaluation tools are essential when planning didactic activities that are respectful of the psycho-physical characteristics of their reference target: (1) Who evaluates: the teacher, the educator, the trainer; (2) Where: the place where the evaluation is taking place (classroom, gym, sports playground); (3) Tools: sets of tests or technological instruments used by the evaluators and other tools used by those who perform "a motor action"; (4) What to assess: based on the evaluator's professional background (a sport trainer, a pre-school or primary school teacher), object of the assessment could be the functional anatomical biomechanical aspects or the cognitive ones such as the acquisition of a motor skill; (5) When: specific moments during the school year (preferably the beginning of the year, the end of first quarter, the end of year); (6) Method: The selection of the measuring method depends on the age group and its anthropometric properties, the observation of the movement executed or the effects that the movement has produced.

\section{Conclusions}

The use of motor evaluation tests in educational contexts includes the regular collection and interpretation of data leading, as integral part of the educational and didactic process, to the definition of a value judgement aimed at an educational action [2]. It is clear that the observation method and the tests, from Meinel's point of view, require that expert teachers or trainers also adopt a series of such indirect methods to set or correct a movement underlining the importance of the choice of tools useful in activating concentration [14].

It is therefore important to prepare and use complementary evaluating tools that can help the 
pre-school teacher to recognize and identify the indicators of the motor repertoire of each pupil already during the first school phases as there is no one gold standard assessment tool to investigate motor ability in children [2].

Among the various batteries of tests, available internationally, related to the quality of motor control in children between 3 and 6 years of age, it is possible to draw a map of the different systems of motor evaluation that are consistent with and functional to the educational offer of the Italian School as well as with the objectives and learning targets outlined in the Italian ministerial guidelines [1, 15].

The Italian context offers batteries of tests, for the cross assessment of motor skills, that are easy to use and widely favoured by the teachers for their adaptable contents and methods. Their aim is to evaluate fine-motor skills, proprioceptive orientation, static-dynamic balance and the praxis skills such as orientation in time and space, dexterity, precision, speed, general and eye-hand coordination.

The study of motor control and in particular of the eye-hand and segmented coordination in children aged between 3 and 6 requires, therefore, a test useful to evaluate: (1) the visual-perceptive skills and the integration of visual-motor skills (developmental test of visual perception tpv); (2) the integration of visual and motor skills (vmi battery-devolpment test of visual-motor integration); (3) the motor difficulties (abc movement battery to evaluate the child's motor system).

A good assessment model supports students' desire to learn. Such an assessment model calls for us as teachers to relinquish some of the control we have held over assessment and give it to the students.

To meet these new demands, a variety of performance-based assessment alternatives have been developed that focus on high-priority objectives and significant outcomes for students. These alternatives are authentic and designed to take place in the real life setting of day-to-day activities in or outside the gym and physical education class, not on artificially contrived "test" days [16, 17].

\section{Reference}

[1] Ministry of Education 2012. Guidelines for the Nursery and Primary School. Roma: Italian Ministry of Education. (in Italian)

[2] Meinel, K. ed. 1991. The Attempt to Form Theories of Sport Movements in Pedagogical Aspects. Roma: Società Stampa Sportiva. (in Italian)

[3] Taylor, K. M., and Sortor, J. M. 2003. "Clinical Value of the BEERY Visual-Motor Integration Supplemental Tests of Visual Perception and Motor Coordination.” Optometry and Vision Science 80 (4): 312-5.

[4] Montessori, M. 2001. Educating for Freedom and Introduction to Pedagogy. Milano: Garzanti. (in Italian)

[5] Bruner, J. 1971. Early Stages of Cognitive Development. Roma: Armando Editore.

[6] Vayer, P. 1968. Psychomotor Education and Mental Retardation. Roma: Armando. (in Italian)

[7] Yu, C., and Smith, L. B. 2013. "Joint Attention without Gaze Following: Human Infants and Their Parents Coordinate Visual Attention to Objects through Eye-Hand Coordination.” PloS One 8 (11): e79659.

[8] Le Boulch, J. 1990. Educational Sport. Roma: Armando editore. (In Italian)

[9] Gardner, H. 2009. Formae Mentis. Essay on Intelligence Plurality. Milano: Garzanti. (in Italian)

[10] Berthoz, A. 1998. The Sense of Movement. Ed. Odile Jacob. (in Italian)

[11] Maude, P. 2001. Physical Children, Active Teaching. Investigating Physical Literacy. Buckingham: Open University Press.

[12] Copple, C., and Bredekamp, S. 2009. Developmentally Appropriate Practice. Washington, DC: National Association for the Education of Young Children.

[13] Goodway, J. D., Wall, S., and Getchell, N. 2009. "Promoting and 'Active Start' for Young Children: Developing Competent and Confident Early Movers.” Strategies 23 (2): 30-2.

[14] Gardner, H. 1998. Educating to Understand. Milano: Feltrinelli. (in Italian)

[15] Piek, J. P., Hands, B., and Licari, M. K. 2012. "Assessment of Motor Functioning in the Preschool Period.” Neuropsychology Review 22 (4): 402-13.

[16] Cools, W., De Martelaer, K., Samaey, C., and Andries, C. 2009. "Movement Skill Assessment of Typically Developing Preschool Children: A Review of Seven Movement Skill Assessment Tools.” Journal of Sports Science and Medicine 8 (2): 154-68. 
[17] Graham, G., Shirley, A. and Parker, M. 1998. Children Moving: A Reflective Approach to Teaching Physical
Education. Mountain View: Mayfield Publishing Company. 